\title{
Décadrages Décadrages
}

cinéma, à travers champs Cinéma, à travers champs

$28 \mid 2014$

Arnaud Desplechin

\section{Festival International du film de Locarno 2014, bilan de la $67^{\circledR}$ édition}

\section{Achilleas Papakonstantis}

\section{(2) OpenEdition}

1 Journals

Édition électronique

URL : https://journals.openedition.org/decadrages/769

DOI : $10.4000 /$ decadrages.769

ISSN : 2297-5977

Éditeur

Association Décadrages

Édition imprimée

Date de publication : 10 octobre 2014

Pagination : 96-109

ISBN : 978-2-9700668-9-7

ISSN : 2235-7823

Référence électronique

Achilleas Papakonstantis, «Festival International du film de Locarno 2014, bilan de la $67^{e}$ édition », Décadrages [En ligne], 28 | 2014, mis en ligne le 11 janvier 2016, consulté le 03 avril 2022. URL : http:// journals.openedition.org/decadrages/769; DOI : https://doi.org/10.4000/decadrages.769 


\section{Festival International du film de Locarno 2014, bilan de la $67^{\mathrm{e}}$ édition}

1 Dans un communiqué daté du I2 août, le réalisateur a renoncé à sa participation au festival par crainte des tensions et des controverses. Polanski aurait dû présenter la projection de $L a$ Vénus à la fourrure le I4 août sur la Piazza Grande et donner une master class le lendemain. Depuis le début du festival, les réactions contre la présence du cinéaste étaient nombreuses et venaient de plusieurs élus suisses. Le député Fiorenzo Dado, du Parti démocrate-chrétien, avait écrit sur Facebook le 6 août: «Aujourd'hui s'ouvre un festival qui honore un pédophile». Ensuite, l'Union Démocratique du Centre a incité ses députés à ne pas participer à la cérémonie de remise d'un Léopard spécial au réalisateur de Rosemary's Baby et Chinatown. Toutes ces réactions se sont référées aux accusations de pédophilie qui accompagnent Polanksi depuis I977 et l'affaire «Samantha Geimer». Le directeur artistique du festival, Carlo Chatrian, a exprimé son amertume dans une vidéo en insistant sur le rôle important du festival en tant que «lieu d'accueil et espace de liberté».

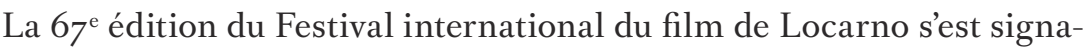
lée par une tendance à faire cohabiter la production indépendante et un cinéma plus récréatif, notamment en programmant plusieurs films «rassembleurs» sur la Piazza Grande (Lucy de Luc Besson, Sils Maria d'Olivier Assayas, Geronimo de Tony Gatlif, Pause de Mathieu Urfer). Cette édition a également signalé le retour dynamique de la politique dans le champ cinématographique, que ce soit par le choix des films (Kommunisten de Jean-Marie Straub, Socialismi de Peter von Bagh, L'Abri de Fernand Melgar, Durak de Yury Bykov, A Blast de Syllas Tzoumerkas, parmi d'autres) ou par des polémiques imprévues culminant avec l'«affaire Polanski» ${ }^{1}$, que les médias suisses et internationaux n'ont pas manqué de relayer. Pourtant, le festival commence peu à peu à trouver son propre équilibre sans souffrir d'un manque d'éclat: si Polanski a renoncé à sa participation à la dernière minute, des personnalités aussi diverses qu'Alexandre Sokourov, Victor Erice, Agnès Varda, Jean-Pierre Léaud, Dario Argento, Juliette Binoche, Oliver Assayas, Mia Farrow, Tony Gatlif, Giancarlo Giannini ou encore Melanie Griffith ont fait le déplacement.

L'audace et la fulgurance ont également caractérisé le concours international (avec Cavalo Dinheiro de Pedro Costa, Mula sa kung ano ang noon de Lav Diaz, The Iron Ministry de J. P. Siadnecki), malgré le choix du jury de récompenser prioritairement des cinéastes découverts dans les éditions précédentes du festival (Lav Diaz, Alex Ross Perry). Au vu de l'étendue du programme, nous avons pris le parti d'accorder une place prioritaire à la production suisse, plus dynamique qu'on a coutume de le dire, tout en abordant certains films internationaux dont la présence a contribué au prestige artistique et à l'identité cinéphilique forte du festival. 


\section{FILMS SUISSES}

Une adaptation originale de Homo Faber

Homo Faber (Trois Femmes) (Richard Dindo, $\mathrm{CH}, 2014$ )

Projeté dans la sélection Fuori Concorso, le dernier long métrage de Richard Dindo s'est imposé comme l'une des ouvres les plus audacieuses du programme (à l'instar des films de Godard et de Straub). Adaptation du célèbre roman homonyme de Max Frisch ${ }^{2}$, Homo Faber (Trois Femmes) constitue une tentative radicale de transposition audiovisuelle d'un texte qui se caractérise par sa focalisation interne ${ }^{3}$. Dindo joue avec différentes possibilités d'énonciation du cinéma dans un film qui exhibe ses modalités d'expression tant sur le plan de la bande image que sur celui de la bande son.

Le personnage principal, Walter Faber, n’apparaît jamais à l'écran. Dindo propose une visualisation des interactions du héros avec les trois femmes qui ont marqué sa vie (Ivy, Hannah et Sabeth), en affirmant la subjectivité de l'image à travers une abondance de déictiques, dont le regard à la caméra est l'exemple le plus récurrent. Pourtant, et contrairement à ce que l'on pourrait croire, on n'assiste pas à des plans subjectifs stricto sensu, soit à des plans vus par les yeux du héros ${ }^{4}$. Faber est un voyageur qui parcourt le monde la caméra en bandoulière, en filmant ses aventures et ses rencontres ${ }^{5}$. En effet, le film de Dindo est composé d'images supposément enregistrées par l'objectif de prise de vue de Faber, auquel le spectateur s’identifie. Cependant, il arrive souvent que la caméra (de Dindo cette fois) se libère des contraintes diégétiques: en dehors de l'intervention récurrente des images fixes et de leur statut

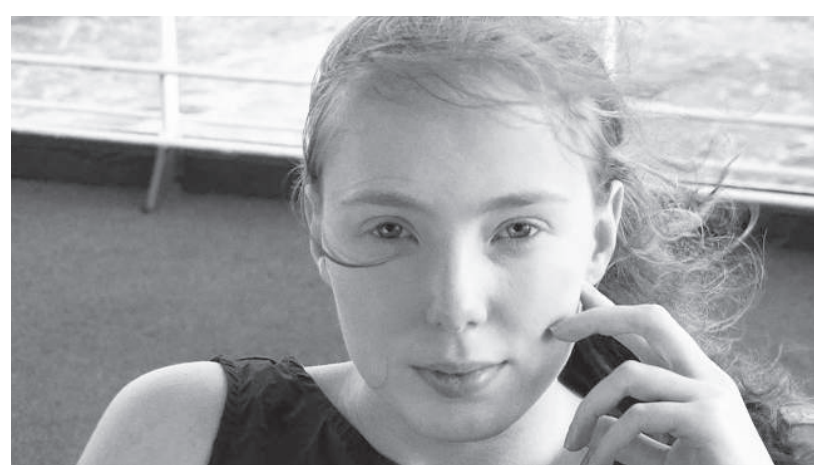

Homo Faber (Trois Femmes) de Richard Dindo
2 Voir Max Frisch, Homo Faber, Paris, Gallimard, i96i [1957]. En i99i, Volker Schlöndorff a livré une première adaptation cinématographique avec Sam Shepard et Julie Delpy dans les rôles principaux. Dindo, pour sa part, s'est déjà attaqué à l'œuvre de l'écrivain suisse dans Max Frisch, Journal I-III, en I98I.

3 «Adapter un texte littéraire écrit au 〈je〉 a toujours constitué une sorte de défi pour les cinéastes, étant donné leur dispositif de captation mécanique qui induit un type d'énonciation que Christian Metz a appelée ‘impersonnelle»» (Valentine Robert, «Le Scaphandre et le papillon et l'adaptation filmique du «je» littéraire: l'œil qui écrit», Décadrages, nº 16 I7, hiver 20I0, p. I07).

4 Quant à l'emploi systématique de la caméra subjective, l'exemple canonique reste le film Lady in the Lake (La Dame du lac, Robert Montgomery, 1947).

5 Cette fonction du héros en tant qu'«opérateur de prises de vue» est également présente dans le roman. Dans ses premières cinquante pages, nous avons compté huit références à l'acte de filmer (mieux, d’avoir filmé) les événements racontés. Par exemple: «Quand la lune se leva (ce que j’ai également filmé) entre les agaves noirs à l'horizon» (Max Frisch, op. cit., p. 28), ou «Il n'y avait absolument rien à filmer» (Max Frisch, id., p. 32) ou encore «Même pour filmer, j’étais trop paresseux» (id., p. 49). 
6 En fait, on est confronté à l'irruption du son diégétique à deux moments dans le film, et seulement pour quelques secondes, en écoutant Sabeth pleurer $\left(47^{\mathrm{e}} \mathrm{min}\right)$ ou rire $\left(80^{\mathrm{e}} \mathrm{min}\right)$. Cette rupture dans la réalité muette de la diégèse, outre son impact émotionnel (suggérant qu'à ces deux moments le souvenir est trop fort pour rester emprisonné dans le passé), peut évoquer, certes sous des modalités différentes, l'irruption momentanée du mouvement dans La Jetée (Chris Marker, i962).

7 Alain Boillat, Du bonimenteur à la voix-over, Lausanne, Antipodes, 2007, p. 326 .

8 Il est intéressant de noter que, selon le générique de fin, nous n'aurions pas affaire à un film, mais à une «lecture cinématographique».

9 Le terme, proposé par François Jost, se réfère à l'assimilation exacte de l'œil de la caméra au regard du personnage (voir François Jost, L'OEil-caméra. Entre film et roman, Lyon, PUL, I987). Dans le cas de Homo Faber, le personnage principal est soit la caméra dont la présence est diégétisée, soit Walter Faber qui regarde constamment à travers le viseur de son appareil.

10 Selon l'analyse d'Alain Boillat, la voix over est un procédé qui donne l'impression au spectateur que le narrateur homodiégétique se rapproche du niveau premier de l'énonciation filmique (Alain Boillat, op. cit., pp. 38I-402). L'exemple le plus caractéristique est l'obéissance apparente du montage au texte proféré par cette voix. L'approche de Dindo dans Homo Faber est plus radicale: le locuteur diégétique est frontalement reconnu comme le «méga-narrateur», comme celui qui a filmé et monté les images projetées.

ambigu (concrétisation instantanée du flux de mémoire de Faber ou affirmation du contrôle de l'énonciateur sur le déroulement temporel du récit visuel), l'exemple le plus saillant intervient lorsque le héros embrasse Sabeth et que l'appareil de prise de vue, assimilé momentanément à la tête de Faber, se penche afin de toucher les lèvres de la jeune fille.

Sur le plan sonore, Homo Faber évince toute trace acoustique provenant de l'univers diégétique ${ }^{6}$. Dans un retour aux modalités du cinéma muet, les actrices improvisent des rôles dénués de parole tandis que Walter Faber fonctionne comme un bonimenteur invisible dont le commentaire profondément nostalgique médiatise notre accès aux événements actualisés par l'image. Nous n'avons toutefois pas affaire à un monologue intérieur; sa voix désincarnée provient d'un moment postérieur aux images, leur assignant une place dans le passé, à savoir son passé, le passé même de cette voix. Ainsi, accompagnée d'une musique extradiégétique, la voix-over à la première personne entraîne l'identification physique du spectateur qui, interpellé, se reconnaît comme le destinataire des paroles du héros. Cette voix acousmatique, qui «implique non seulement un espace virtuel [...] mais aussi et surtout une certaine temporalité: la présence se perçoit au présent " ${ }^{7}$, parcourt l'ensemble du film. Par conséquent, le spectateur se trouve lié au narrateur grâce à sa contemporanéité (au «maintenant» de son émission vocale) contrairement aux images qui demeurent, elles, éloignées, cantonnées dans le passé par le langage verbal.

Dindo exploite tous les paradoxes induits par un narrateur homodiégétique, voire auto-diégétique, constamment relégué hors-champ; il résout ainsi le problème de l'audiovisualisation du «je» littéraire en transposant l'écriture à travers l'acte de filmer et de commenter a posteriori les images projetées ${ }^{8}$. Au croisement des procédés de la voix over et d'une ocularisation interne primaire ${ }^{9}$, la présence de l'énonciateur est directement thématisée ${ }^{10}$ (et, malgré son invisibilité, diégétisée) à tel point que le film se présente dans son ensemble comme la confession poignante d'un «méga-narrateur » ${ }^{11}$. L'exclusion du protagoniste par le cadrage (que

11 «Le monstrateur-narrateur filmique, véritable méga-narrateur [...] réaliserait donc en syncrétisme l'union, la fusion des deux modes fondamentaux de la communication narrative: la monstration et la narration.» (André Gaudreault, Du littéraire au filmique. Système du récit, Paris, Nota Bene, I999, p. I07). 
lui-même détermine) exacerbe la douleur de ses souvenirs et lui rappelle la distance inaltérable qui le sépare d'Ivy, d'Hannah et de Sabeth ${ }^{12}$. Par sa dimension autoréflexive, Homo Faber évite les pièges exhibitionnistes tendus par la subjectivité de l'image et atteint un effet immersif en réaffirmant le postulat cher aux Cahiers du cinéma des années i970 selon lequel toute fiction constitue un documentaire sur son propre tournage.

\section{Une certaine tendance du cinéma documentaire suisse}

Mon père, la révolution et moi (Ufuk Emiroglu, $\mathrm{CH}, 2014$ )

L'Abri (Fernand Melgar, CH, 2014)

Je suis Femen (Alain Margot $\mathrm{CH}, 2014$ )

Broken Land (Stéphanie Barbey et Luc Peter, CH, 2014)

Si le documentaire occupe depuis longtemps une place d'honneur dans la production cinématographique suisse, la sélection offerte par la $67^{\mathrm{e}}$ édition du Festival de Locarno ne se limite pas à la construction d'une mémoire collective du pays. En effet, les quatre films sur lesquels nous nous proposons de revenir engagent un concept géographique plus vaste et affichent un caractère international marqué, que ce soit par le biais du sujet de l'immigration à l'intérieur du territoire helvétique (Mon père, la révolution et moi et L'Abri), ou par l'intérêt porté à des phénomènes allogènes aux échos helvétiques explicites (le passage des activistes du mouvement «Femen» par la Suisse) ou symboliques (la problématique des frontières dans Broken Land quelques mois seulement après l'acceptation par les Suisses de l'initiative du 9 février 2014 restreignant la libre circulation des personnes). Si la question politique est fortement présente, nous assistons néanmoins à une tentative de la part des réalisateurs de se distancer soigneusement de toute intention militante. Parmi les quatre films présentés, certains réalisateurs se contentent de porter quelques combats à la connaissance des spectateurs, tandis que d'autres cherchent plus ambitieusement à développer une réflexion sur une réalité donnée; tous se retrouvent pourtant confrontés aux enjeux éthiques et politiques que tout documentaire soulève.

Mon père, la révolution et moi adopte la formule du journal intime, inscrite dans un régime de confession autobiographique sans aucune volonté de mise à distance. Pourtant, l'histoire familiale d'Emiroglu présente des implications politiques extraordinaires qui nous emmènent de la Turquie des années I970 vers la Suisse du XXI ${ }^{\mathrm{e}}$ siècle. Malheureusement,
12 Dans les dernières minutes du film, tandis que Sabeth fixe du regard le spectateur, Faber répète les mots suivants à son propos: «ce visage qui n'est plus, ses yeux qui ne sont plus, son corps qui n'est plus». Tout se passe comme si le personnage voulait désespérément croire aux théories ontologiques baziniennes et au pouvoir qu'a l'image photographique de maintenir en vie la jeune fille décédée et son amour passé. 
13 L'hétérogénéité des formats est impressionnante: on y rencontre des home videos, des images d'animation, d'archive, ou encore des séquences fictionnalisées et reconstituées par des acteurs.

14 Comme, par exemple, l'autoproclamée "ville libre de Christiania» à Copenhague, rassemblant une communauté libertaire et autogérée possédant son propre drapeau et sa propre monnaie.

15 Voir le schéma proposé dans JeanMichel Adam, Le Récit, Paris, PUF, 1984.

16 Pour une analyse du film, voir Marthe Porret, "Un goût sucré d'asile», dans Décadrages, $\mathrm{n}^{\circ} \mathrm{I} 3$, automne 2008, pp. I02-IO4; Séverine Graff, «Sans populisme, ni militantisme. Représentation du migrant dans La Forteresse de Melgar», dans Décadrages, $\mathrm{n}^{\circ} \mathrm{I} 4$, printemps 2009, pp. 39-50.

17 L'intervention de Melgar prend une ampleur toute particulière à Locarno puisque la ville a voté «oui» à l'initiative «Contre l'immigration de masse» à un taux supérieur à $64 \%$. le croisement entre la vie personnelle et les événements historiques qui la traversent est maladroitement traité; la réalisatrice privilégie son imaginaire au réel historique, qui est traité de façon spectaculaire. La mixité des images ${ }^{13}$, rassemblées au sein d'une comédie loufoque, témoigne du désir d'esthétisation d'un discours politique empreint de raccourcis argumentatifs: une commémoration stérile des activités de la gauche en Turquie d'il y a trente ou quarante ans, un rejet trop rapide de différentes communautés contemporaines qui échappent aux normes sociopolitiques actuelles ${ }^{14}$ et la glorification finale du mode de vie suisse en fournissent des exemples éloquents.

Tout au long du film, les cicatrices de l'Histoire ne laissent transparaître qu'une blessure narcissique, qui témoigne de l'espoir d'Emiroglu d'exorciser ses démons intérieurs. En tant que narratrice-énonciatrice, elle s'affirme par l'usage récurrent du «je» en voix over dont le ton intimiste vise à instaurer une relation privilégiée avec le spectateur. Son commentaire, loin d'être neutre, apporte une interprétation personnelle d'événements historico-politiques tout en sollicitant la complicité du spectateur par le biais de l'ironie et de la parodie. Par ailleurs, nous avons affaire à un documentaire caractérisé par une dramaturgie de type fictionnel classique (situation initiale - complication - résolution situation finale) ${ }^{15}$, tandis que la construction du personnage/narrateur central est conforme aux codes du récit d'initiation postadolescent, très répandu dans le cinéma de fiction dominant. Ainsi, Mon père, la révolution et moi se caractérise par un discours complaisant qui tend à dénier toute responsabilité politique au film. Nous sommes loin du mouvement protestataire de la place Taksim à Istanbul.

De l'attribution du Léopard d'or de la catégorie «Cinéastes du présent» pour La Forteresse (2008) ${ }^{16}$ à la dénonciation de Vol spécial (20II), qualifié d'«opus fasciste» par le président du jury, Paulo Branco, Fernand Melgar peut témoigner des deux pôles opposés de la réception locarnaise. Dans ce contexte, les réactions modérées et quasi-unanimes suscitées cette année par son dernier documentaire, L’Abri, sélectionné en compétition internationale, s'accomodent à un film moins audacieux que les précédents. Ceci malgré une prise de parole ouvertement polémique du réalisateur avant la projection de son film - clamant son indignation devant le resurgissement de la xénophobie en Suisse, manifesté par la dernière votation contre l'immigration ${ }^{17}$. Melgar s'intéresse encore une 


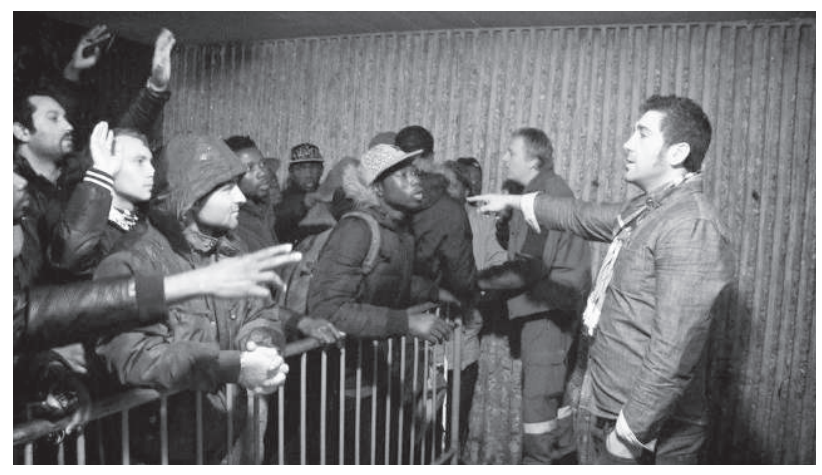

L'Abri de Fernand Melgar fois aux populations en marge de la société (composées majoritairement de migrants économiques) et, par sa visée informative, L’Abri émerge comme un document important (voire nécessaire) qui dévoile une réalité derrière l'apparence aseptisée de l'espace public lausannois.

Tourné à l'abri PC de la Vallée de la Jeunesse à Lausanne, le film témoigne de la vie quotidienne de dizaines d'hommes, de femmes et d'enfants qui, privés d'hébergement d'urgence par manque de place, sont contraints de dormir dans la rue, même en plein hiver. Parallèlement, nous assistons à la routine du travail des veilleurs de l'abri qui, chaque nuit, doivent trier les pauvres et décider de qui aura droit ou non à un lit et à un repas chaud. Il est vrai que Melgar n’a jamais réalisé de simples «reportages»; son cinéma, basé sur l'individualisation progressive de cas représentatifs, exprime un milieu de l'intérieur en amenant ses personnages à se mettre eux-mêmes en scène. Il s'agit effectivement d'une approche délicate et pas aussi impersonnelle qu'elle pourrait sembler l'être à première vue. Le souci du partage équilibré du temps à l'écran des deux pôles de son récit (d'une part les sans-domicile, d'autre part les employés de l'abri et, notamment, parmi eux, José) témoigne du désir d'inféoder le récit à un principe de neutralité, accentué par l'effacement de l'instance d'énonciation filmique.

Cependant, donner une voix propre à chaque personne portraiturée n'est pas une tâche facile lorsqu'il s'agit pour Melgar de mettre en scène plusieurs groupes aux statuts très inégaux. Les écarts socioculturels ne sont pas pris en compte et sa prétendue position d'observateur objectif ne l'empêchent pas de faire appel à un imaginaire entourant la vie des 
18 Une analyse approfondie du film devrait également prendre en compte des aspects parafilmiques, comme par exemple la présence de José et du directeur de l'abri aux côtés de Melgar lors de la conférence de presse du festival. «sans-domicile», voire à un univers parallèle que le spectateur peine à imaginer. Ce dernier (à l'instar du cinéaste lui-même) se sent de connivence avec José, le veilleur au grand cœur. A ce point, il est intéressant de noter une différence stratégique entre La Forteresse et L'Abri. Dans le premier film, le récit individualise le responsable du Centre d'accueil des requérants d'asile, soit une personne qui se différencie nettement du spectateur par sa position institutionnelle et, donc, qui peut plus facilement devenir la cible d'une critique strictement sociopolitique. En revanche, dans L'Abri, le personnage singularisé (José) est un simple employé dont les intentions bienveillantes se dessinent clairement via les conflits, présentés de manière réitérée et parfois comique, qui l'opposent au responsable de l'abri. Le portrait de ce dernier, cynique et caricatural, le rend antipathique et permet ainsi de sortir de la salle la conscience tranquille, puisque son personnage cristallise les problèmes que le film expose. L'efficacité du film est dès lors atténuée, L'Abri montrant malgré lui la façon dont un souci humanitaire se solde finalement par une indifférence politique ${ }^{18}$.

Je suis Femen, quant à lui, est une chronique du groupe homonyme ouvertement sympathisante à sa cause. Déjà présenté aux Visions du réel à Nyon en avril et en avant-première à Lausanne en mai 20I4, le documentaire d'Alain Margot est, selon sa productrice Caroline Velan, un film sur le courage et la jeunesse, voire le portrait glorifiant d'une jeune femme qui «voulait changer le monde». Oxanna Shachko, artiste spécialisée dans la peinture d'icônes religieuses, a cofondé avec Anna Hutsol et les sœurs Inna et Sasha Schevchenko en Ukraine le groupe Femen, participant à la dynamisation de la scène des luttes féministes au XXI ${ }^{\mathrm{e}}$ siècle, même s'il ne fait pas l'unanimité au sein des mouvements militants. Avec un penchant assez prononcé pour la provocation, cette «communauté» ouverte et étrangère à toute tendance sectaire ne présente aucune réticence à étendre son champ de bataille: pour les orphelins et les animaux maltraités, contre le patriarcat ou le tournoi de football européen 2012 tenu en Pologne et en Ukraine, ou encore contre Timochenko et Poutine.

Ce sont également avec des expressions auto-satisfaites qu'Oxanna et ses camarades relatent leurs accomplissements. Cette forme d'éloquence rend d'emblée superflu un commentaire explicatif; Margot laisse donc la parole et l'initiative à ses protagonistes, soit par des entretiens face caméra, soit par des scènes prises sur le vif (manifestations, affrontements avec la police, etc.). Or ce choix empêche le cinéaste de pénétrer en profondeur 
dans les retentissements d'une activité militante telle que celle engagée par Femen, notamment par rapport aux frontières entre la médiatisation et l'institutionnalisation d'un mouvement de contre-pouvoir ou aux potentialités subversives de l'image du corps nu. Ceci le conduit souvent à des explications psychologiques discutables, comme lorsque qu'il associe l'absence d'une figure paternelle au fondement de l'action féministe d'Oxanna. De plus, l'esthétisation à la limite de l'exhibitionnisme s'avère une tentation que Margot n'évite pas, comme en témoignent les effets graphiques du générique d'ouverture ou l'emploi ponctuel du ralenti. Finalement, Je suis Femen reste classique dans sa forme et ne se démarque pas des représentations canoniques mais, en optant pour un positionnement clair envers son sujet, gagne en efficacité discursive face à une tendance inquiétante de pseudo-neutralité qui se manifeste dans le cinéma documentaire suisse présenté lors de cette édition du festival.

Parmi les films regroupés ici, Broken Land est le seul à proposer, de manière consciente et stimulante, une véritable mise à distance face aux événements traités. Projeté en première mondiale dans la sélection Semaine de la critique, le documentaire de Stéphanie Barbey et Luc Peter nous amène dans un territoire désertique de l'Arizona, à la frontière entre les Etats-Unis et le Mexique, où une petite communauté d'Américains vit dans l'ombre du mur érigé afin de limiter l'immigration clandestine. De lents panoramiques, parfois contemplatifs, dévoilent un paysage aride et font appel à l'imaginaire du western classique tout en le détournant: le mythe d'une frontière pleine de promesses s'avère n'être finalement qu'un leurre. Le couple de réalisateurs étonne par l'humilité

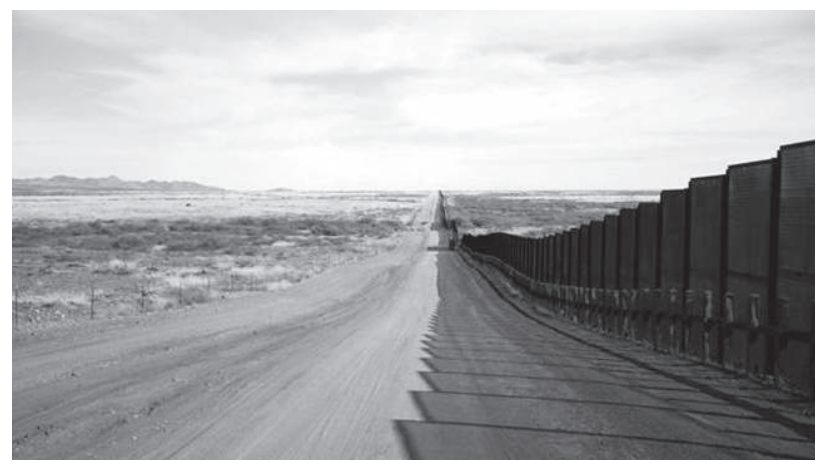


de leur approche. Sans défendre des positions préétablies, les cinéastes posent des questions et enregistrent les réponses avec la distance nécessaire pour que les contradictions inhérentes aux propos des personnes interviewées surgissent par elles-mêmes dans toute leur ampleur.

Contrairement à l'approche de Melgar, Barbey et Peter reconnaissent l'inégalité de statut entre les groupes représentés, à savoir le décalage qui sépare d'emblée Mexicains et Etasuniens. Ainsi, les premiers n'apparaissent dans le film que comme des silhouettes ombreuses captées par des caméras de surveillance: des fantômes dont la présence bouleverse la vie des seconds qui sont contraints de sortir de leur passivité et de se positionner face à ce phénomène de migration. Broken Land offre une variété de points de vue qui se développent à l'intérieur de la communauté américaine et qui peuvent éventuellement s'entrechoquer grâce à la structure paratactique du récit qui juxtapose des épisodes distincts. La simplicité de la mise en scène, accompagnée discrètement par la musique de Franz Treichler (leader du groupe Young Gods), contribue à un discours débarrassé de tout sentimentalisme. Un spectateur attentif appréciera le positionnement politique des cinéastes: l'image d'un chat qui hésite à franchir une barrière - la tête au Mexique et la queue sur le sol des Etats-Unis - ou celle d'un berger allemand qui observe avec grand intérêt un groupe de poussins pendant que leur maître, garde-frontière américain, remarque avec déception que ses compatriotes sont «trop gentils» envers les menaces extérieures.

\section{Les jeux interdits d'Andrea Štaka}

Cure - The Life of Another (Andrea Štaka, Suisse/Croatie/ Bosnie-Herzégovine, 2014).

Récompensée par un Léopard d'or pour son premier long métrage (Das Fräulein) en 2006, Andrea Štaka était de retour cette année dans le concours international du festival de Locarno. Cure - The Life of Another partage avec le film précédent un même motif central, à savoir les enjeux identitaires de femmes de générations différentes sous le coup de la guerre en ex-Yougoslavie. En 1993, Linda, moitié Suisse et moitié Croate, accompagne son père de retour dans sa ville natale de Dubrovnik. A l'école, elle rencontre Eta qui devient rapidement sa meilleure amie. Après leurs cours, les deux jeunes adolescentes se rendent ensemble dans une forêt au bord de la mer. Là-bas, elles sadonnent à des jeux de 
provocation, chargés de sous-entendus sexuels, qui s'interrompent brusquement avec la chute mortelle d'Eta. Štaka escamote alors l'intrigue policière de son film afin de suivre la figure énigmatique de Linda, qui prend peu à peu la place d'Eta dans sa famille.

L'idée d'un meurtre inavoué, voire refoulé, et de rédemption par un jeu de substitution (de la victime par son double) se prêterait facilement à un thriller psychologique hitchcockien dans le sillage de Vertigo ${ }^{19}$. Heureusement, la réalisatrice zurichoise s'intéresse pour sa part aux connotations symboliques de cette affaire policière et construit une allégorie de la guerre aux allures fortement autobiographiques. Si le film fonctionne si bien, malgré son histoire peu originale, c'est principalement grâce au rôle primordial accordé au décor naturel qu'offre la ville de Dubrovnik. La beauté plastique du paysage coexiste et contraste fortement avec les atrocités commises sur les champs de bataille, communiquées exclusivement par le son lointain mais persistant des bombardements. Cette ambiance suscite auprès des personnages principaux (Linda, la mère et la grand-mère d'Eta) un sentiment inéluctable d'impuissance; trois femmes à différentes étapes de leur vie sont prises au cœur d'un monde dont le sens leur échappe. La guerre devient ainsi une crise de l'intersubjectivité qui défait les identités, dès lors brisées et insaisissables. Mais Cure n'est pas dénué de facilités, notamment quant aux choix stylistiques exhibés (effets de miroir récurrents, irruptions d'inserts analeptiques ou encore apparitions fantomatiques) qui font flirter le récit avec l'irréel, entraînant par là une surcharge de type métaphysique, atténué pourtant par la qualité du jeu de ses interprètes.

\section{Bande à part: Godard et Straub à Locarno}

Adieu au langage (Jean-Luc Godard, France, 2014)

Kommunisten (Jean-Marie Straub, Suisse, 2014)

Si Locarno s'attache traditionnellement à la découverte de nouveaux talents, il s'avère que les films les plus visionnaires de cette édition $2014 \mathrm{du}$ festival ont été signés par deux réalisateurs vétérans. A première vue, le rapprochement entre un cinéaste qui, déjà par son choix de titre, rumine la possibilité d'une séparation définitive avec le langage et un autre dont la carrière est marquée de manière quasi-obsessionnelle par la matérialité de la langue peut sembler paradoxal. A part une camaraderie qui date des années I960 et une référence spatiale commune (la ville de Rolle ${ }^{20}$ ),
19 Film d'Alfred Hitchcock de 1958 avec James Stewart et Kim Novak.

20 Même si Adieu au langage est une production française, nous l'incluons dans la partie consacrée aux films suisses projetés au festival, au vu du rôle central que la ville de Rolle et le paysage lémanique entretiennent avec ce film. 
21 Le titre d'un court métrage réalisé par Straub et Huillet en I977, adapté du poème de Stephane Mallarmé.

22 Pour la reconstitution et l'analyse du dispositif godardien, voir Stefan Kristensen, Jean-Luc Godard philosophe, Lausanne, L’Age d'Homme, 20I4.

23 L'inclusion du spectateur dans le dispositif godardien n'est pas une affaire récente. Voir par exemple les propos du cinéaste à l'époque de Numéro Deux: «Au cinéma actuellement, ce sont les spectateurs qui créent les films. [...] C'est [le spectateur] qui relie l'image d'avant à celle d'après. [...] C'est le spectateur qui fait le boulot», Entretien avec Jean-Luc Godard dans Libération, $\mathrm{n}^{\circ} 2$, $15 \mathrm{sep}-$ tembre 1975 , repris dans Godard par Godard, Paris, Editions de l'Etoile / Cahiers du Cinéma, I985, p. 38I.

Adieu au langage de Jean-Luc Godard
Godard et Straub se rencontrent cette année grâce à la place qu’ils accordent au spectateur à l'intérieur de leur dispositif. Devant leurs derniers films, nous sommes bien ailleurs que dans une simple séance de cinéma; effectivement, nous sommes invités à créer notre propre film, dans un rituel à la fois mélancolique et jubilatoire, comme des «enfants qui jouent aux trois dés $(3 \mathrm{D})$ » (un des nombreux jeux de mots présents dans le film de Godard) puisque «toute révolution est un coup de dés» ${ }^{21}$.

Le public locarnais a eu l'opportunité de découvrir, trois mois après sa première projection au festival de Cannes, le dernier film de Jean-Luc Godard, Adieu au langage. La 32, comme c'était le cas avec la technologie vidéo au début des années I970, prend sa place dans le dispositif godardien ${ }^{22}$ et témoigne de la volonté persistante du cinéaste de mâ̂triser techniquement les nouveaux médias pour mieux les critiquer de l'intérieur. Une place spécifique est assignée au spectateur qui, renvoyé à sa singularité et non plus traité comme une entité interchangeable (la notion abstraite de "public», voire de «consommateur»), est appelé à effectuer son propre montage et à participer au dévoilement des rapports (de force) qui sous-tendent la production audiovisuelle ${ }^{23}$. Ainsi, Adieu au langage se déploie sur huit pistes simultanées, six sonores et deux pour l'image, et nous sommes invités à participer au processus de montage et de mixage à travers des gestes physiologiques, voire à imaginer d'autres possibilités de relations qui peuvent se nouer entre les éléments du film. Certaines séquences reposant sur une disjonction totale des images, que le spectateur «monte» à son gré en fermant l'œil gauche ou l'œil droit, en fournissent un exemple éloquent.

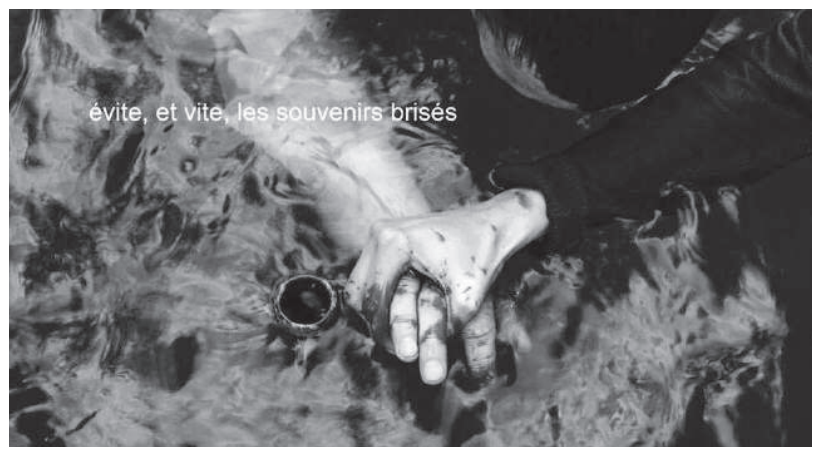


Le dernier opus de Jean-Marie Straub, quant à lui, est arrivé à Locarno inachevé. Mais si les producteurs de Kommunisten ont pris le soin de préciser aux festivaliers qu'ils n'assistaient qu'à une version de travail, cette qualification ne saurait réduire l'ampleur politique et l'ambition éthique d'un film qui dépasse de loin le simple exercice de l'autocitation. Certes, il propose un retour en arrière, un voyage sélectif dans la filmographie de son auteur; parmi les six chapitres, seul le premier (Le Temps du mépris dont le tournage a eu lieu à Rolle en juillet 20I4) est véritablement nouveau. Le reste du film se compose d'extraits tirés de l'œuvre du couple Straub-Huillet - dans l'ordre: Ouvriers, paysans (200I), Trop tôt, trop tard (1982), Fortini/Cani (1976), La Mort d'Empédocle (1987) et Noir péché (1989). Mais, ici comme ailleurs, l'acte autocitationnel constitue une procédure à travers laquelle le film s'affirme en tant que tel, condition sine qua non pour parvenir à «l'être-en-cinéma » ${ }^{24}$, à savoir le grand projet de toute l'œuvre des Straub. Cet être-en-cinéma ne renvoie pas à un espace métaphysique et lointain; il est bien au contraire inexorablement lié à la matière dont l'exacerbation se réalise dans le film de manière polyvalente: par l'omniprésence de la nature dans ses manifestations concrètes (du mouvement des feuilles au jeu de cache-cache entre ombre et lumière, entraîné par des nuages cantonnés dans le hors-champ), par l'affichage du travail des acteurs (loin des conventions psychologiques et des présupposés mythologiques), mais encore par la structure globale qui relève d'une construction géométrique (reliant un ensemble hétérogène d'images, de textes et de langues). Kommunisten constitue donc un véritable travail de récapitulation, celle du cinéma et de sa capacité à exprimer de manière objective ${ }^{25}$, fidèle à la matière, une idée politique qui, comme un spectre, continue à hanter le monde.

\section{PRODUCTION INTERNATIONALE \\ Contes contemporains en temps de crise}

Cavalo Dinheiro (Pedro Costa, Portugal, 2014)

Durak (Yury Bykov, Russie, 2014)

A Blast (Syllas Tzoumerkas, Grèce/Allemagne/Hollande, 2014)

Récompensé à juste titre par le Prix de la mise en scène, le dernier opus de Pedro Costa offre une coda élégiaque au projet qu'on pourrait désormais désigner comme la «tétralogie de Fontainhas» ${ }^{26}$. Cavalo Dinheiro retrouve Ventura, figure emblématique de ce quartier délabré de Lisbonne,
24 Même si les Straub repartent presque exclusivement dans leur filmographie d'œuvres littéraires ou musicales, leur réflexion s'exprime à travers leurs outils, à savoir les images, les sons et leur articulation. Cet effort que Philippe Lafosse appelle "être-en-cinéma» fait écho aux propos de Deleuze sur l'importance pour les cinéastes d'avoir au moins une idée en cinéma. Voir Philippe Lafosse, L'Etrange cas de Madame Huillet et Monsieur Straub, Toulouse/Ivry-sur-Seine, Editions Ombres / A propos, 2007, p. I6.

25 A propos de la recherche de l'objectivité radicale dans le cinéma de StraubHuillet, voir Benoît Turquety, Danièle Huillet et Jean-Marie Straub. "Objectivistes» en cinéma, Lausanne, L'Age d'Homme, 2009.

26 Les trois premières parties sont Ossos (I997), No Quarto da Vanda (2000) et Juventude em Marcha (2006). 


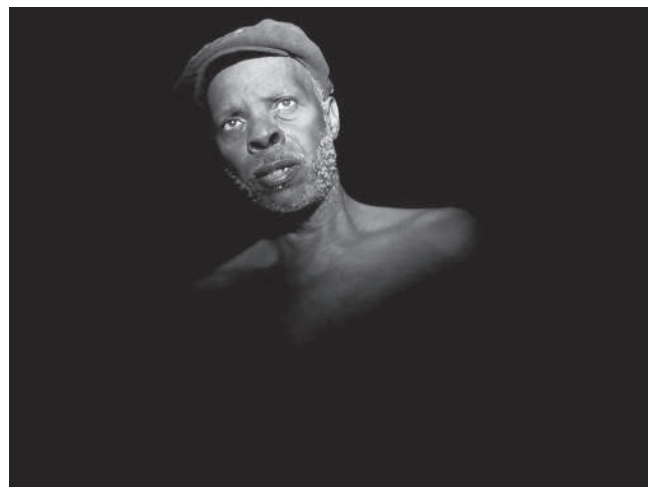

27 Il nous faut distinguer les termes «durak» (дурак) et «idiot» (Идиот) dans la langue russe. Le premier, traduit littéralement par «niais» ou «simplet», fait référence à un personnage folklorique qui, malgré ses capacités mentales inférieures, gagne la sympathie du lecteur/ spectateur par son caractère «têtu». En revanche, le second (employé par Dostoïevski dans le titre de son célèbre roman) présente des connotations plus tragiques et moins sarcastiques. Malheureusement, le titre international du film (The Idiot) ne rend pas justice aux allusions subtiles du titre original. maintenant enfermé dans un hôpital. Le visage balayé de tics et de convulsions, il titube comme un fantôme dans un monde décomposé tendant vers l'abstraction, obtenue grâce au minimalisme du décor. Tiraillé entre pulsions de vie et de mort, Ventura résiste à l'effondrement de tout ce qui l'entoure. Ses yeux ont avalé les plus effroyables visions et les souvenirs font de lui moins un corps vivant qu’un paysage marqué par des luttes infinies. Son rapport au temps, s'il existe encore, repose sur la suspension et les boucles. Le film, largement silencieux mais marqué par une série de monologues fougueux, est indiscutablement habité par la rigueur du cadre. Fidèle au choix pris pendant le tournage d'Ossos d'éviter tant que possible les mouvements de caméra, jugés trop esthétisants voire manipulateurs par nature, Costa opte encore une fois pour un format d’image dur (I.33: I), devenu encore plus sévère par la fixité des plans et l'emploi systématique du «cadre dans le cadre». Cavalo Dinheiro s'affirme comme un geste radical d'un cinéaste au style affirmé qui adresse aux spectateurs sensibles les bruissements les plus subtils.

Si Pedro Costa a inévitablement aliéné une grande partie du public locarnais, c'est un film russe qui a suscité la passion et fait l'unanimité des spectateurs. Durak n'est pas, comme la traduction anglaise de son titre (The Idiot) le ferait croire, une nouvelle adaptation filmique du chef-d'œuvre dostoïevskien ${ }^{27}$. En revanche il s'agit là d'un portrait nihiliste et profondément politique de la corruption en Russie au XXI ${ }^{\mathrm{e}}$ siècle. Le comique se mêle au tragique, le caractère saugrenu des situations représentées n'est jamais loin, tandis que l'ensemble entre en résonance - avec une justesse 
et une précision inquiétante - avec les souffrances contemporaines d'un grand nombre de peuples européens. Le récit suit la nuit de suspense d'un jeune ingénieur qui s'évertue à sauver la vie d'environ huit cents habitants d'un bâtiment de logements sociaux en train de s'effondrer. Dans sa course, il se perd dans les méandres d'un système politique gangréné et subit la structure pyramidale d'une société hiérarchisée, dont les seules valeurs semblent être le profit et l'exploitation de l'autre. Malgré quelques scènes trop esthétisantes, comme l'interlude musical, filmé en travelling latéral, qui arrive de manière complètement inattendue au milieu du film, Yuri Bykov excelle à mêler comédie noire, satire politique et thriller de compte à rebours. Il a par ailleurs la chance de s'appuyer sur un jeune interprète remarquable, Artem Bystrov, récompensé en fin de festival.

A l'instar de Durak, le nouveau film de Syllas Tzoumerkas, A Blast, est également marqué par le sentiment d'un chaos imminent et inéluctable. Pour le jeune cinéaste grec, la crise sociale, politique et culturelle est affaire d'adrénaline; son récit, pluricentré et foudroyant, tient le spectateur en haleine. L'implacable attraction du mal dans une société corrompue est le prétexte d'un drame déconcertant à l'atmosphère viciée, centré sur une famille au bord de l'hystérie. Cependant, la confrontation symbolique entre générations - des jeunes confrontés aux dettes de leurs parents - est ancrée dans des éléments plus prosaïques (voir par exemple l'irresponsabilité fiscale de la mère acariâtre et autarcique qui demeure mal justifiée au niveau dramaturgique) qui témoignent d'une interprétation naïve et superficielle de la crise qui frappe la Grèce. Au-delà de la rudesse des situations et malgré une direction d'acteurs impressionnante, le dérèglement des comportements des personnages principaux ne conduit pas à une confusion et un chaos de gestes et de voix qui auraient pu, comme dans le cinéma de Yannis Economides (Soul Kicking, Knifer, Stratos), illuminer une réalité sociale cruelle. Ainsi, l'univers diégétique est habité par une galerie d'abrutis dont le cinéaste se distancie nettement - on est d'ailleurs dans une focalisation zéro et les analepses récurrentes ne sont imputées à aucun personnage. Mais l'incongruité la plus frappante de A Blast repose sur ce penchant regrettable du nouveau cinéma grec pour l'excentricité exagérée des personnages: prendre une exception pour en faire le centre du film sans suggérer qu'il s'agit d'une exception, c'est l'ériger en règle. 